DOE/ER/40561-21-INT98

LBNL-42172

\title{
Enhancement of Intermediate Mass Dileptons From Charm Decays At SPS Energies
}

\author{
Ziwei Lin and Xin-Nian Wang \\ Nuclear Science Division, LBNL, Berkeley, CA 94720 \\ and \\ Institute for Nuclear Theory, University of Washington, Box 351550, Seattle, WA 98195
}

December 31, 2017

\begin{abstract}
We study the dimuon yield from open charm decays in the intermediate mass region(IMR) in heavy-ion collisions at the CERN-SPS energy. We find that final state rescatterings which broaden the $m_{T}$ spectrum of charmed mesons can enrich the part of the phase space covered by the NA50 experiment, thus leading to an apparent enhancement of IMR dileptons within the acceptance of the experiment. Such an enhancement increases with the dimuon invariant mass and the single muon energy cut-off.
\end{abstract}

Dileptons are important signals in high-energy heavy-ion collisions. Depending on their invariant masses they can probe the collision processes at different stages. Low invariantmass dileptons are predominantly produced at the late stage of the collisions. So they provide information about the medium modification of hadron properties such as mass and spectral functions [1]:2]. A large enhancement of low invariant-mass dileptons has indeed been 
observed in HELIOS-3 [3] and CERES [4] experiments. Many theoretical studies attributed this enhancement either to a dropping $\rho$-mass in a medium of high baryon-density [5], or to the collisional broadening of hadronic spectral functions [6].

At large invariant masses, dileptons are mostly produced from partonic processes in the early stage of heavy-ion collisions. For example, dileptons from the Drell-Yan processes [7] dominate the large invariant mass region at the CERN-SPS energy. Thus they can provide a benchmark for parton luminosity or the centrality of the collisions in which proposed signals of the phase transition to the quark-gluon plasma such as $J / \psi$ suppression [8] and thermal dileptons [9] can be studied via the dilepton channel. However, at collider energies such as the BNL RHIC and CERN LHC, the Drell-Yan processes no longer dominate dilepton productions at reasonably large invariant masses. Dileptons from decays of charm and bottom mesons instead dominate [10]. These large invariant-mass dileptons from heavy quark decays may also give us information about energy loss of heavy quarks traveling through the dense medium 11$] 13$.

As one can naively expect, both partonic and hadronic processes will contribute to dileptons with invariant masses between the above noted regions, i.e., in the intermediate mass region (IMR). An enhancement of intermediate-mass dileptons was observed in $S+W$ collisions at HELIOS-3 [3] and was attributed to secondary meson-meson interactions by Gale and Li [14. NA38/NA50 experiments [15,16] observed similar enhancement in the mass region $1.5 \mathrm{GeV} / \mathrm{c}^{2}<M_{\mu^{+} \mu^{-}}<2.5 \mathrm{GeV} / \mathrm{c}^{2}$. The dimuon invariant-mass spectrum in $p+p$ collisions is fitted by a combination of dileptons from Drell-Yan and decays of charmed mesons. Assuming a scaling of binary collisions for hard processes, it was found that dimuon spectra in $p+A$ collisions can be well accounted for but results from $S+U$ and especially $\mathrm{Pb}+\mathrm{Pb}$ collisions indicate significant enhancement [16,17]. It is difficult to argue that the excess of IMR dileptons comes from enhanced charm production according to previous estimates [18,19] in which it is concluded that the charm production from secondary parton scattering is negligible with a realistic estimate of temperature and gluon fugacity at both RHIC and LHC energies. Even if a quark-gluon plasma could be formed at the CERN-SPS 
energy, the temperature and gluon fugacity are probably too low to produce any significant amount of charmed quarks. The charm production in a hadronic gas can also be neglected.

We focus in this paper on another possible scenario in which IMR dileptons can be enhanced in the acceptance of NA50 $P b+P b$ experiment. We demonstrate that the modification of the phase space distribution of D-mesons due to final state rescatterings can change the shape of the dilepton distribution in phase space. Since most experiments only measure a small part of the whole phase space, the redistribution of the dileptons from decays of charmed mesons can give rise to an apparent enhancement of IMR dileptons within the limited acceptance of the experiments.

In the dense medium formed during heavy-ion collisions, interactions among hadrons or partons will lead to partial thermalization of the system. Thus, effects of strong final state rescatterings are expected, e.g., in the experimentally measured particle spectra in transverse momentum [20]. These spectra can be parameterized in an exponential form, $d N / m_{T} d m_{T} \propto e^{-\left(m_{T}-m\right) / T_{e f f}}$, and are characterized by the inverse slope $T_{e f f}$. It is found that $T_{\text {eff }}$ increases with the system size [21,22] and is roughly a linear function of the particle mass. For example, $T_{\text {eff }}$ for protons increases from $148 \mathrm{MeV}$ in $p p$ collisions to 208 $\mathrm{MeV}$ in central $S S$ collisions, and to $289 \mathrm{MeV}$ in central $P b+P b$ collisions at measured by NA44 23]. The increase is often attributed to a collective transverse flow, which essentially comes from final state rescatterings among secondary particles [24]. Such effect of final state rescatterings which causes the broadening of hadronic $m_{T}$ spectra, could also happen to $D$-mesons $\left(D^{0}, \bar{D}^{0}, D^{+}, D^{-}\right.$etc $)$.

To obtain a qualitative feature of this effect, we model final state rescatterings in the following way. We assume that D-mesons thermalize with their local environment, where the degree of thermalization is parameterized by a temperature $T$. Due to the lack of knowledge about interactions between D-mesons and other hadrons, one cannot accurately estimate the value of $T$. We assume that D-meson spectrum is influenced by final state rescatterings in the same way as other hadrons. Thus, $T_{\text {eff }}$ for D-mesons in central $P b+P b$ collisions is enhanced to about the same value as those for protons and $\phi$-mesons $(290 \mathrm{MeV})$. From this 
$T_{\text {eff }}$, the parameter $T$ can be constrained to be about $150 \mathrm{MeV}$ in our model. Note that $T$ is the local temperature which differs from the definition of $T_{\text {eff }}$. Later in the discussion we will show the dependence of our results on $T$.

We calculate the open charm production in $p p$ collisions with the latest PYTHIA program [25]. We use the MRS D-' [26] parton distribution functions with intrinsic parton transverse momentum $k_{t}$ of $1 \mathrm{GeV} / \mathrm{c}$, and with $m_{c}=1.3 \mathrm{GeV} / \mathrm{c}^{2}$. The charm pair cross section in $p p$ collisions, $\sigma_{c \bar{c}}^{p p}$, is normalized to $9.5 \pm 2 \mu \mathrm{b}$ at $E_{L a b}=200 \mathrm{GeV}$ [15], which then gives $6.8 \mu \mathrm{b}$ at $E_{L a b}=158 \mathrm{GeV}$. For central $\mathrm{Pb}+\mathrm{Pb}$ collisions at $158 \mathrm{AGeV}$, the number of charm pairs is then given by

$$
N_{c \bar{c}}^{P b P b}=\sigma_{c \bar{c}}^{p p} T_{P b P b}(0),
$$

where the overlap function $T_{P b P b}(0)$ is taken to be $30.4 / \mathrm{mb}$ [27].

We take the Peterson fragmentation function to describe the hadronization of charmed quarks to D-mesons:

$$
D(z) \propto \frac{1}{z[1-1 / z-\epsilon /(1-z)]^{2}}
$$

where $z=p_{D} / p_{c}$, and $\epsilon=0.06$. D-mesons are then assumed to be thermalized in its local frame with a temperature parameter $T$ as the following. For a D-meson with an initial four-momentum $p_{D, \alpha}=\left(E_{D}, \vec{p}_{D}\right)$ in the Lab frame, first a local random momentum in the D-meson rest frame is generated from the thermal distribution $d N / d^{3} p_{D}^{l} \propto e^{-E_{D}^{l} / T}$. Then the local four-momentum $\left(E_{D}^{l}, \vec{p}_{D}\right)$ is boosted back to the Lab frame, i.e., to the frame with $\gamma_{D}=1 / \sqrt{1-\beta_{D}^{2}}$, where $\vec{\beta}_{D}=\vec{p}_{D} / E_{D}$. This will simulate a locally thermal D-meson spectrum with some collective flow in both transverse and longitudinal directions. The local temperature $T$ is taken to be $150 \mathrm{MeV}$ if not specified otherwise in this paper.

Shown in Fig.1 are the $m_{T}$ spectra within $-0.5<y_{D}<0.5$ and the rapidity distribution for initial and final D-mesons. All figures are normalized to one central $P b+P b$ event at $E_{L a b}=158 \mathrm{AGeV}$. As expected, initial and final D-mesons have roughly the same rapidity spectra, and the inverse slope of the $m_{T}$ spectra at small values of $\left(m_{T}-m_{D}\right)$ increases 
significantly after the inclusion of the simulated final state rescatterings. The inverse slope parameter, $T_{\text {eff }}$, corresponding to a fit in the region $\left(m_{T}-m_{D}\right) \in(0,1) \mathrm{GeV} / \mathrm{c}^{2}$ increases from $160 \mathrm{MeV}$ to $290 \mathrm{MeV}$. To show the correlation of charm and anti-charm particles in a pair, we plot the azimuthal angle difference, the rapidity difference between the two and the correlation in $\cos \theta_{C S}$ in Fig.2, where $\cos \theta_{C S}$ is the angle of one $\mathrm{D}$ meson relative to the beam axis in the rest frame of the charm pair. The correlation in azimuthal angles is reduced by random scatterings, however, the rapidity gap between the two particles in a charmed meson pair remains almost the same. The distribution in $\cos \theta_{C S}$ would be flat if charm mesons would follow a static-fireball distribution so that there would be no correlation between two charm mesons in a pair. However, the charmed meson pair are still strongly correlated in rapidity with a big rapidity gap in our model due to the simulated longitudinal flow. This is the reason why there is still a strong correlation in $\cos \theta_{C S}$ despite of final state rescatterings. In fact, the correlation in $\cos \theta_{C S}$ still remains strong even if one removes the initial correlation in azimuthal angles, $\phi_{12}$, of the charmed meson pair.

For semileptonic decays of D-mesons to muons, the average branching ratio of $c \rightarrow \mu^{+} X$ is taken to be $12 \%$. The muon energy spectrum from $D$-meson decays is consistent with the measurement of the MARK-III experiment [28]. Since the number of charm pairs, $N_{c \bar{c}}^{P b P}$, is much less than 1, the dilepton spectrum from decays of uncorrelated charm pairs is negligible. In addition, we note that like-sign subtraction is applied in the NA50 data analysis.

The dimuon invariant-mass, pair rapidity, and $\theta_{C S}^{\mu}$, which is the angle of one muon relative to the beam axis in the dimuon rest frame(Collins-Soper reference frame), are defined as:

$$
\begin{aligned}
M_{\mu^{+} \mu^{-}} & =\sqrt{\left(p_{\alpha}^{\mu^{+}}+p_{\alpha}^{\mu^{-}}\right)^{2}} \\
Y_{\mu^{+} \mu^{-}} & =\tanh ^{-1} \beta_{z, \text { pair }} \\
\cos \theta_{C S}^{\mu^{+}} & =\frac{p_{z}^{\mu^{+}}-\beta_{z, \text { pair }} E^{\mu^{+}}}{\sqrt{1-\beta_{z, \text { pair }}^{2}} \sqrt{M_{\mu^{+} \mu^{-}}^{2} / 4-m_{\mu}^{2}}} .
\end{aligned}
$$

where $\beta_{z, \text { pair }}=\left(p_{z}^{\mu^{+}}+p_{z}^{\mu^{-}}\right) /\left(E^{\mu^{+}}+E^{\mu^{-}}\right)$.

Invariant-mass spectra of dimuons from charm pair decays are plotted in Fig. 3. The total 
dimuon yield does not change since the total charm yield does not change. However, there are much more high invariant-mass dimuons after final state rescatterings. The dimuon pair rapidity distribution has little change.

To consider the effect of the limited acceptance of an experiment, we approximate the NA50 acceptance by taking the following cuts on dimuon variables:

$$
M_{\mu^{+} \mu^{-}}>1.5 \mathrm{GeV} / \mathrm{c}^{2}, Y_{\mu^{+} \mu^{-}}^{C M S} \in(0 ., 1 .), \cos \theta_{C S}^{\mu^{+}} \in(-0.5,0.5)
$$

and cuts on single muons:

$$
\begin{array}{rlrl}
\theta^{\mu} & \in(0.037,0.108), & & \\
E^{\mu}>8 .+1600 \times\left(\theta^{\mu}-0.065\right)^{2}, \text { when } \theta^{\mu} & \in(0.037,0.065), \\
& >8 ., & & \in(0.065,0.090), \\
& >8 .+1300 \times\left(\theta^{\mu}-0.090\right)^{2}, & & \in(0.090,0.108),
\end{array}
$$

where $Y_{\mu^{+} \mu^{-}}^{C M S}=Y_{\mu^{+} \mu^{-}}-2.9$ for $E_{L a b}=158 \mathrm{AGeV}$.

The invariant-mass, pair rapidity and $\cos \theta_{C S}^{\mu^{+}}$spectra inside this NA50 acceptance are shown in Fig. 4 . Due to the broadening of the D-meson $m_{T}$ spectrum, the dimuon yield from D-meson decays in the above acceptance is enhanced significantly. The enhancement is stronger at larger dimuon invariant masses, reflecting the relative change of the $m_{T}$ spectrum. However, the shapes of the pair rapidity spectrum and the $\cos \theta_{C S}^{\mu^{+}}$spectrum stay roughly the same. We define the enhancement factor of the dimuon yield from open charm decays inside the acceptance, $R$, as the ratio between accepted dimuon yields after and before final state rescatterings. It is equivalent to the area under the solid curves divided by the area under the dashed curves in Fig. 4 $\mathrm{b}$ and $\mathrm{c}$. With a value of $T=150 \mathrm{MeV}$ for final state rescatterings which corresponds to a D-meson $m_{T}$ inverse slope of $290 \mathrm{MeV}$, one obtains an enhancement factor about $R=3.0$.

As we have demonstrated so far, the enhancement factor of IMR dimuons from charmed meson decays, $R$, depends on the D-meson transverse momentum spectrum characterized by $T_{\text {eff }}$. This inverse slope is related to the parameter $T$, which in our model controls the 
strength of final state rescatterings. As one can easily imagine that the degree of final state rescatterings must depend on the size of the dense matter in heavy-ion collisions which in turn depends on the impact parameter. In principle one can study the effect of rescatterings in a cascade model and study the dependence of the effective $T_{e f f}$ or the enhancement factor $R$ on the centrality of heavy-ion collisions. To demonstrate such possible dependence, we simply vary the effective local temperature $T$ here and study how the effective IMR dilepton spectra will change. Show in Fig.5 are the final D-meson $m_{T}$ spectra for different values of temperature $T$. The inverse slopes, $T_{\text {eff }}$, from these spectra can also be read off in Fig. 6, where the relations $R\left(T_{\text {eff }}\right)$ and $T\left(T_{e f f}\right)$ are shown. The two boxes on the left in Fig. 6 refer to the expected dimuon yield by simply scaling up $p p$ results without consideration of final state rescatterings, thus $T=0$ and $R=1$ by definition. The two boxes on the right refer to the enhanced dimuon yield due to final state rescatterings with a default value of $T=150$ $\mathrm{MeV}$. Both the $R\left(T_{\text {eff }}\right)$ and $T\left(T_{\text {eff }}\right)$ relation are roughly linear, thus the $R(T)$ relation is also almost linear. However, the relation between $T$ or $T_{\text {eff }}$ and the impact parameter could be more complex, which we will not address in this paper.

We would like to point out that the absolute value of the total charm cross section, $\sigma_{c \bar{c}}^{p p}$, is not important for our study of the enhancement factor, $R$, for dimuons from charmed meson decays; because we normalize $R$ to 1 for $p A$ collisions and thus $\sigma_{c \bar{c}}^{p p}$ cancels. We take $\sigma_{c \bar{c}}^{p p}$ to be $6.8 \mu \mathrm{b}$ just to be consistent with the NA38 data [15.

Besides final state rescatterings, there is also some effect from initial multiple scatterings, which effectively increases the parton intrinsic $k_{t}$. By modeling initial multiple scatterings in a random-walk picture where the additional average $k_{t}^{2}$ is proportional to the system size, we checked that this effect gives a dimuon enhancement less than 10\%. Anti-shadowing could be a source for the enhancement too, because at SPS energies the charm production probes the region with Bjorken $x \sim 0.2$, where the gluon anti-shadowing could be close to its maximum value [29]. However, this effect is also checked to be no more than $20 \%$.

In summary, the effect of final state rescatterings on D-mesons in $\mathrm{Pb}+\mathrm{Pb}$ collisions at SPS energies are studied. We found that the dimuon yield from open charm decays inside the 
NA50 acceptance is significantly enhanced as a result. Our model for the effects of final state rescatterings is very schematic. However, the essential point is the broadening of D-meson $m_{T}$ spectra, which can be roughly represented by the increase of $T_{\text {eff }}$, the inverse slope of the D-meson $m_{T}$ spectrum. More studies can be done beyond this schematic model. The centrality dependence of the enhancement can be studied and compared to experimental data at various values of total $E_{T}$. The $p_{\perp}$ dependence of the enhancement can also be studied by applying various $p_{\perp}$ cuts, and a larger enhancement is expected when more stringent energy cut is applied to single muons. However, the ultimate test of this model should come from direct measurements of D-meson spectra.

Acknowledgments: We are grateful to the Institute for Nuclear Theory at the University of Washington for supporting the INT-98-1 program where this work was started. Special thanks are due to C. Gale for numerous valuable discussions during the program. We also thank C. Lourenço, K. Redlich, H. Satz, E. Scomparin and N. Xu for helpful discussions. 


\section{FIGURES}
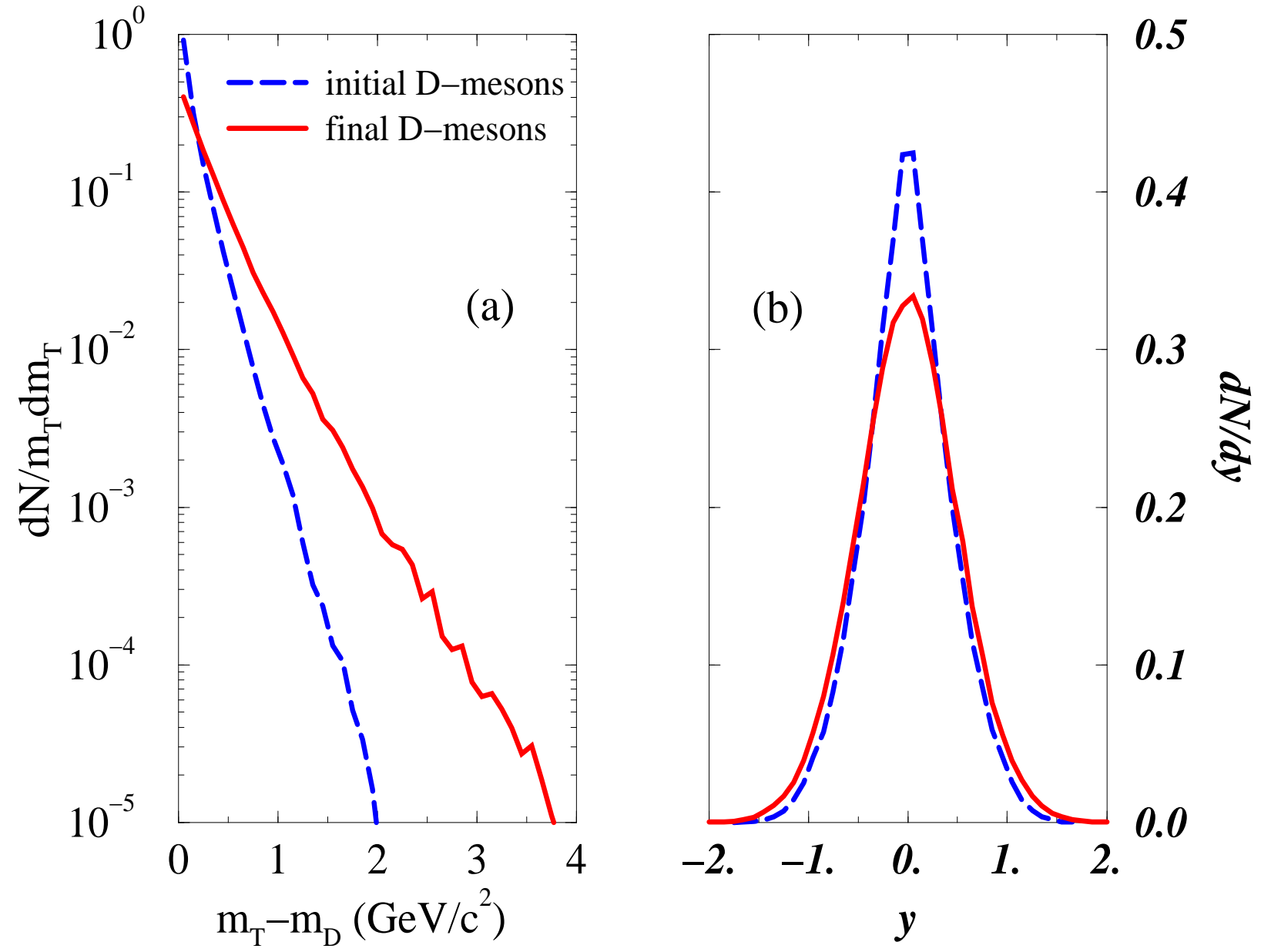

FIG. 1. (a) $m_{T}$ spectra of initial and final D-mesons at mid-rapidity. (b) Rapidity spectra of initial and final D-mesons in the CMS frame. The dashed curve represents initial D-mesons after the Peterson fragmentation, and the solid curve represents final D-mesons after the thermalization with $T=150 \mathrm{MeV}$. 


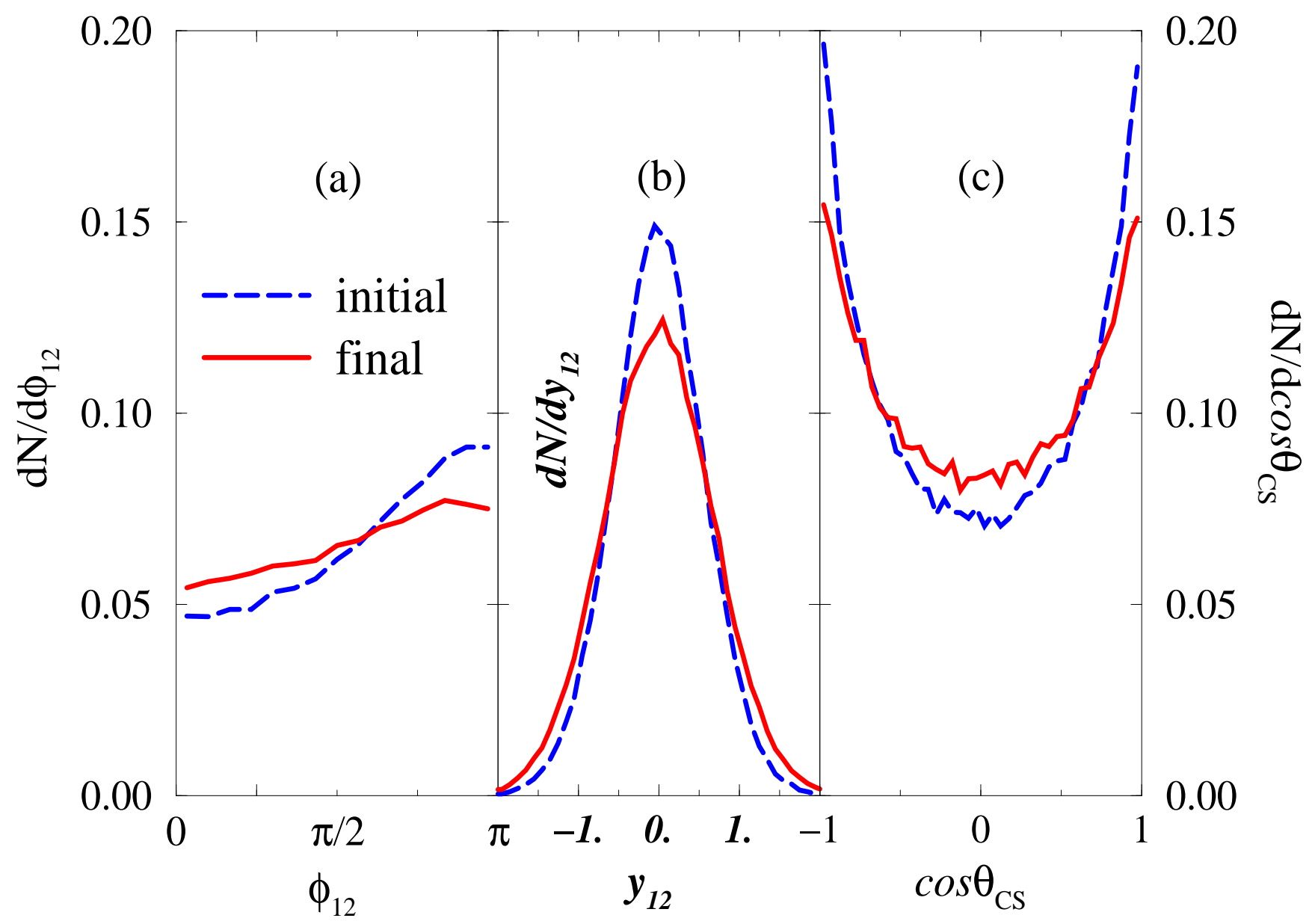

FIG. 2. Spectra (a) as a function of $\phi_{12}$, the difference between the two azimuthal angles; (b) as a function of $y_{12}$, the difference between the two rapidities; and (c) as a function of $\cos \theta_{C S}$, of a charmed meson pair. 


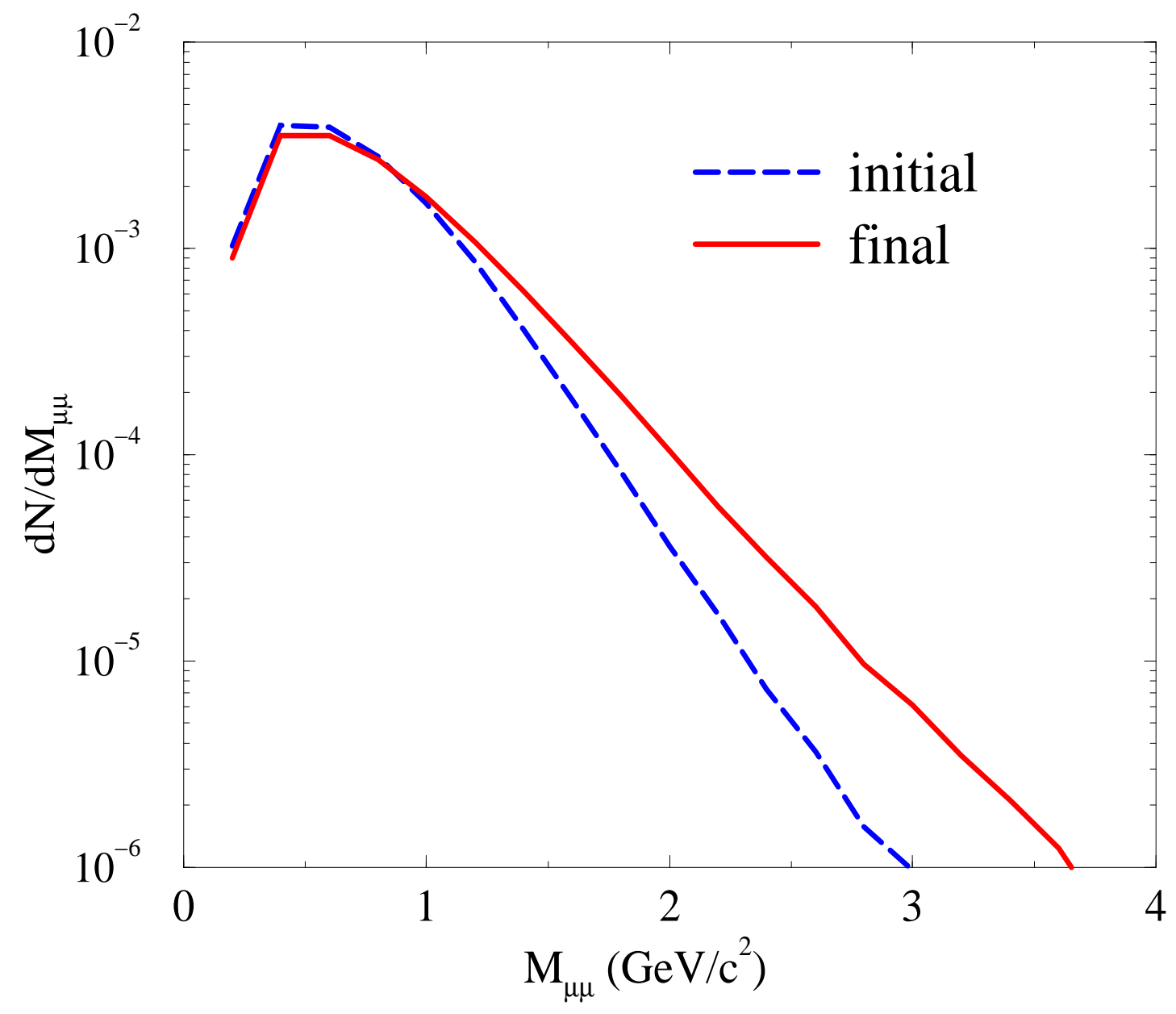

FIG. 3. Invariant-mass spectra of dimuons from charm pair decays. The dashed and solid curves come from D-meson decays without and with final state rescatterings, respectively. 

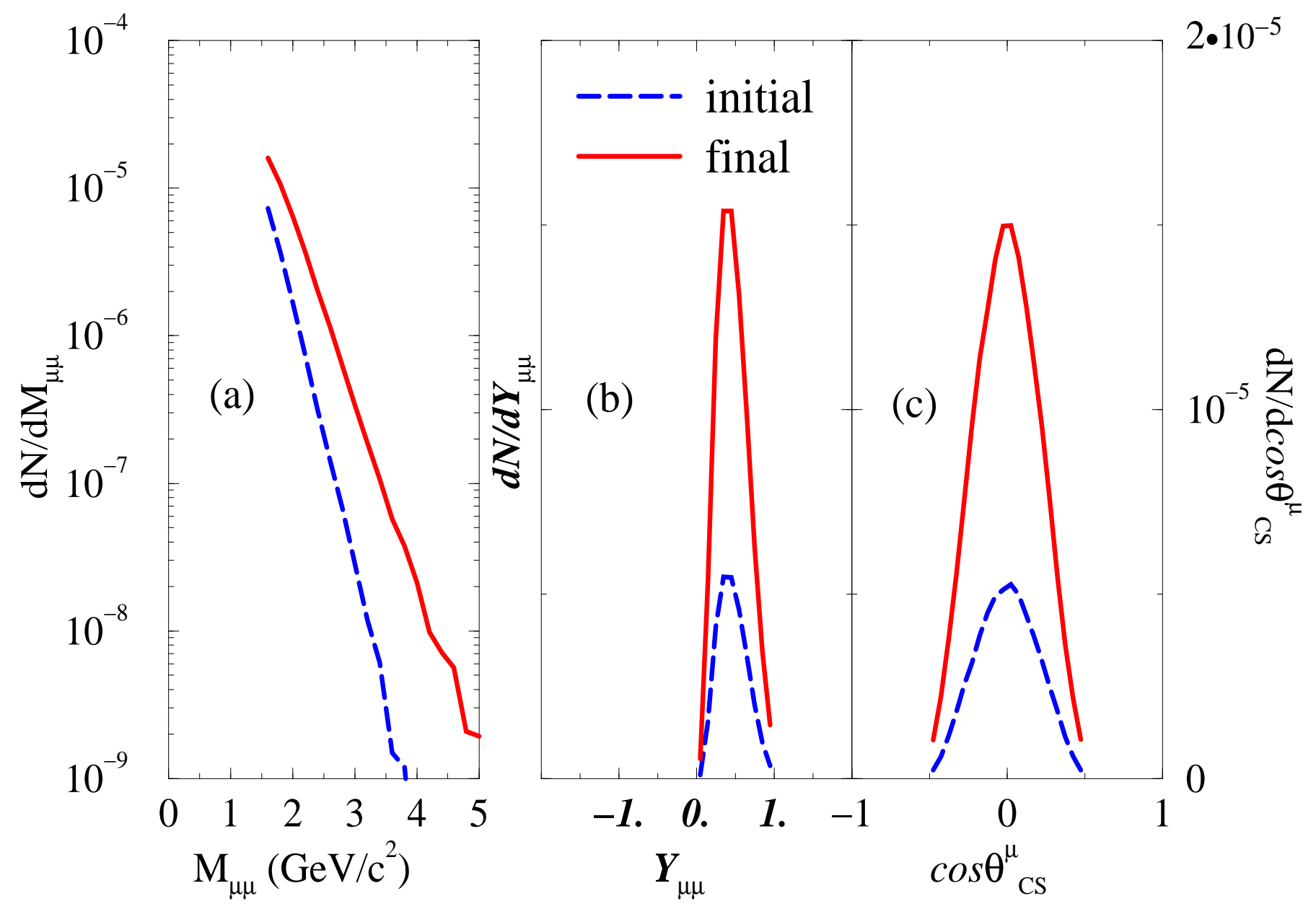

FIG. 4. (a) Invariant-mass, (b) pair rapidity and (c) $\cos \theta_{C S}^{\mu^{+}}$spectra of dimuons from charm pair decays inside the approximate NA50 acceptance. 




FIG. 5. $\quad m_{T}$ spectra of D-mesons at mid-rapidity after different final state rescatterings. 


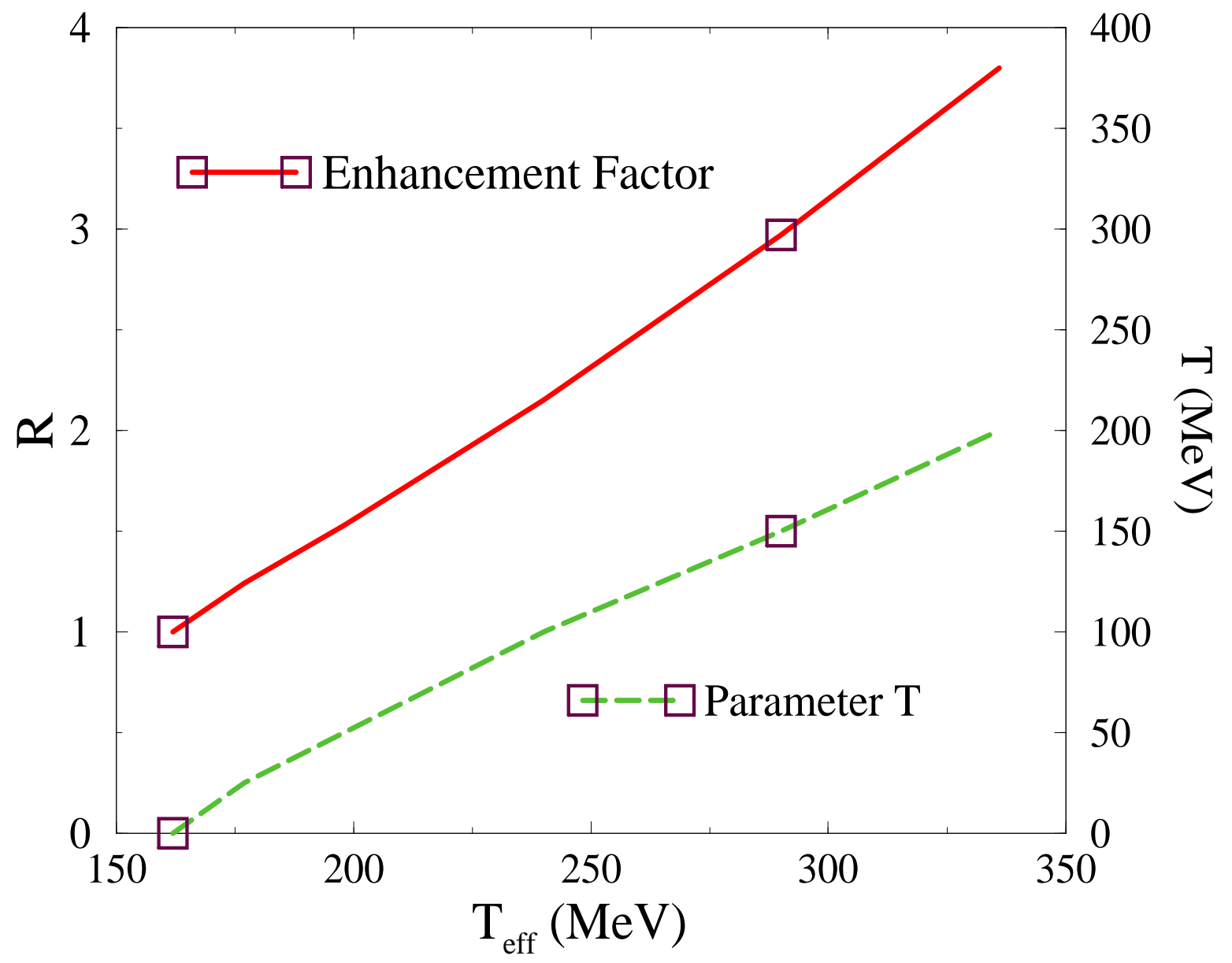

FIG. 6. The dimuon enhancement factor, $R$, and the parameter $T$, as a function of the D-meson inverse slope, $T_{\text {eff }}$. The two boxes on the left refer to the case without final state rescatterings, while the two boxes on the right refer to the default case of final state rescatterings with $T=150$ $\mathrm{MeV}$. 


\section{REFERENCES}

[1] G. E. Brown and M. Rho, Phys. Rev. Lett. 66, 2720 (1991).

[2] T. Hatsuda and S. H. Lee, Phys. Rev. C46, R34 (1992).

[3] M. Masera for the HELIOS-3 Collab., Nucl. Phys. A590, 93c (1995).

[4] A. Drees, Nucl. Phys. A610, 536c (1996).

[5] C. M. Ko, G. Q. Li, G. E. Brown and H. Sorge, Nucl. Phys. A610, 342c (1996).

[6] W. Cassing, E. L. Bratkovskaya, R. Rapp and J. Wambach, Phys. Rev. C57, 916 (1998).

[7] S. D. Drell and T.-M. Yan, Phys. Rev. Lett. 25, 316 (1970); ERRATUM-ibid. 25, 902 $(1970)$.

[8] T. Matsui and H. Satz, Phys. Lett. B178, 416 (1986).

[9] E. Shuryak and L. Xiong, Phys. Rev. Lett. 70, 2241 (1993).

[10] R. Vogt, B. V. Jacak, P. L. McGaughey and P. V. Ruuskanen, Phys. Rev. D49, 3345 (1994).

[11] E. Shuryak, Phys. Rev. C55, 961 (1997).

[12] Z. Lin, R. Vogt and X.-N. Wang, Phys. Rev. C57, 899 (1998).

[13] B. Kämpfer, O.P. Pavlenko and K. Gallmeister, Phys. Lett. B419, 412 (1998).

[14] G. Q. Li and C. Gale, preprint nucl-th/9807005.

[15] C. Lourenço, Ph.D thesis, Universidade Tecnica de Lisboa, 1995;

[16] E. Scomparin for the NA50 Collab., Nucl. Phys. A610, 331c (1996).

[17] E. Scomparin, talk at the INT 1998 Spring Program, University of Washington, 1998.

[18] Z. Lin and M. Gyulassy, Phys. Rev. C51, 2177 (1995); Erratum-ibid. C52, 440 (1995). 
[19] P. Lévai, B. Müller and X.-N. Wang, Phys. Rev. C51, 3326 (1995).

[20] K. S. Lee, U. Heinz and E. Schnedermann, Z. Phys. C48, 525 (1990).

[21] N. Xu for the NA44 Collab., Nucl. Phys. A610, 175c (1996).

[22] G. Roland for the NA49 Collab., Quark Matter'97 contribution.

[23] NA44 Collab., I. G. Bearden et al., Phys. Rev. Lett. 78, 2080 (1997).

[24] J.-Y. Ollitrault, preprint nucl-ex/9802005.

[25] T. Sjöstrand, Comput. Phys. Commun. 82, 74 (1994), on internet see http://nimis.thep.lu.se/tf2/staff/torbjorn/pythia/pythia6115.).

[26] A. D. Martin, W. J. Stirling and R. G. Roberts, Phys. Lett. B306, 145 (1993); ERRATUM-ibid. B309, 492 (1993).

[27] K. J. Eskola, R. Vogt and X.-N. Wang, Int. J. Mod. Phys. A10, 3087 (1995).

[28] MARK-III Collab., R.M. Baltrusaitis et al., Phys. Rev. Lett. 54, 1976 (1985); ERRATUM-ibid. 55, 638 (1985).

[29] K. J. Eskola, V. J. Kolhinen and C. A. Salgado, preprint hep-ph/9807297. 